\title{
Giant lipid vesicles under electric field pulses assessed by non invasive imaging
}

\author{
Chloé Mauroy $^{\mathrm{a}, \mathrm{b}}$, Thomas Portet ${ }^{\mathrm{a}, \mathrm{b}, 1}$, Martin Winterhalder ${ }^{\mathrm{c}}$, Elisabeth Bellard ${ }^{\mathrm{a}, \mathrm{b}}$, Marie-Claire Blache ${ }^{\mathrm{a}, \mathrm{b}, 2}$, \\ Justin Teissié ${ }^{\mathrm{a}, \mathrm{b}}$, Andreas Zumbusch ${ }^{\mathrm{c}}$, Marie-Pierre Rols ${ }^{\mathrm{a}, \mathrm{b}, *}$ \\ a CNRS, IPBS (Institut de Pharmacologie et de Biologie Structurale), BP 64182, 205 route de Narbonne, F-31077 Toulouse, France \\ b Université de Toulouse, UPS, IPBS, F-31077 Toulouse, France \\ c Department of Chemistry, University of Konstanz, Universitätsstraße 10, D-78457 Konstanz, Germany
}

Keywords:

Pulsed electric fields

Electroporation

Imaging

Phospholipids

\begin{abstract}
A B S T R A C T
We present experimental results regarding the effects of electric pulses on giant unilamellar vesicles (GUVs) We have used phase contrast and coherent anti Stokes Raman scattering (CARS) microscopy as relevant optical approaches to gain insight into membrane changes under electropermeabilization. No addition of exogenous molecules (lipid analogue, fluorescent dye) was needed. Therefore, experiments were performed on pure lipid systems avoiding possible artefacts linked to their use. Structural membrane changes were assessed by loss of contrast inside the GUVs due to sucrose and glucose mixing. Our observations, performed at the single vesicle level, indicate these changes are under the control of the number of pulses and field intensity. Larger number of pulses enhances membrane alterations. A threshold value of the field intensity must be applied to allow exchange of molecules between GUVs and the external medium. This threshold depends on the size of the vesicles, the larger GUVs being affected at lower electric field strengths than the smaller ones. Our experimental data are well described by a simple model in which molecule entry is driven by direct exchange. The CARS microscopic study of the effect of pulse duration confirms that pulses, in the ms time range, induce loss of lipids and membrane deformations facing the electrodes.
\end{abstract}

\section{Introduction}

Membranes are key constituents of living organisms, acting as bi ological barriers which hinder the passage of ions and hydrophilic molecules. This selective impermeability is crucial for life to develop. However, these barriers are a hurdle in biotechnological and medical applications where molecule delivery is mandatory. For the purpose of nucleic acids delivery, viral based methods are available. While being quite efficient, their safety has been questioned [1]. Therefore, alternative chemical and physical methods are actively investigated. Such methods, although safer, are less efficient than the viral ones. Among the physical methods, electropermeabilization is one of the most widely used $[2,3]$. Indeed, the cell membrane can efficiently and transiently be permeabilized by applying electric pulses. Provided that

\footnotetext{
* Corresponding author at: CNRS, IPBS (Institut de Pharmacologie et de Biologie Structurale), BP 64182, 205 route de Narbonne, F-31077 Toulouse, France. Tel.: + 33 5611758 11; fax: + 33561175994 .

E-mail address: rols@ipbs.fr (M.-P. Rols).

1 Present address: Department of Chemistry, University of Washington Box 351700 , Seattle, WA 98195-1700, USA.

2 Present address: CIML - Centre d'Immunologie Marseille-Luminy - Parc Scientifique et Technologique de Luminy, 163 avenue de Luminy, Case 906, 13288 Marseille cedex 9, France.
}

the pulses are of a sufficient amplitude and duration, non permanent molecules can enter the cytoplasm of mammalian cells [4]. Because of its efficiency, this method, also referred as "electroporation", is rapidly becoming an established approach for treatment of cancer [5 7] and it also holds great promise for gene therapy [8,9]. If the exact mechanisms of molecular uptake are still poorly understood for the moment, it is known that they depend strongly on the size of the molecules. Whereas smaller molecules freely cross the electropermeabilized membranes, large molecules such as plasmid DNA exhibit the formation of a transient complex with the membrane [10]. Apart from pulse strength, pulse duration is another key parameter for the success of permeabilization, especially in the case of nucleic acids which are driven by electrophoresis towards the permeabilized membrane $[11,12]$. A precise description of the events leading to membrane per meabilization, and its consequences on lipid organization, is still miss ing and would help to design safer and more efficient protocols particularly in the case of gene transfer. However, studying such phe nomena at the molecular level in cells and tissues is currently out of reach. This is a major motivation for investigating the behavior of simpler model systems, namely lipid vesicles.

Synthetic lipid vesicles provide membrane models suitable for sys tematic investigations of the effects of electric fields on lipid bilayers such as electrodeformation, permeabilization and fusion [13,14]. Mem brane permeabilization can be assessed by conductance measurements or transfer of radioactive or fluorescent molecules. Different types of 
vesicles can be used to access membrane electropermeabilization, but only the largest ones (above $10 \mu \mathrm{m}$ ) offer direct visualization of the pro cesses. First experiments have been performed on LUV (15 $30 \mathrm{~nm}$ ) more than 30 years ago [15]. A transient leakage of radioactive sucrose was detected and was attributed to the formation of pores, which allowed passage of sucrose. However, there was no direct proof of their existence, or other related phenomena such as membrane defor mations. GUVs containing fluorescent phospholipid analogs allowed the visualization of membrane associated perturbations under fluores cence microscope [16]. Lipid loss was observed in electropermeabilized membranes and was associated to pore, vesicle, and tubule formation [17]. DNA electrotransfer into liposomes was first studied 20 years ago on LUVs (100 $200 \mathrm{~nm}$ ), suggesting endocytosis like vesicles which shielded the electrotransferred DNA from the internal medium [18]. Yet, also in this case, the liposomes could not be directly observed be cause of their small size. The results were recently questioned by data obtained on giant liposomes supporting a mechanism involving electro pores and a direct entrance of fluorescent DNA into the liposomes [19]. All these results demonstrate that giant vesicles provide a very useful model for approaching the effect of electric field pulses on lipid mem branes because vesicle dynamics can be observed with optical micros copy. However, up to now, visualization of electropermeabilization has been performed by using exogenous fluorescent molecules (lipid analogue, fluorescent dye) and not pure lipid systems thus including possible artefacts linked to these molecules.

The objective of our paper was to perform experiments on pure lipid GUVs, i.e. without any addition of exogenous molecules (lipid analogue, fluorescent dye). For that, we have used two different methods, phase contrast and CARS microscopies, as relevant optical approaches to gain insight into membrane electropermeabilization. GUVs were prepared in a medium containing sucrose and diluted in a medium containing glucose. The difference in refractive index be tween the internal and the external media was detected by phase contrast optics [20]. Such an approach has been described to detect flow out of giant vesicles submitted to electric pulses [21,22]. CARS has the advantage of offering molecular specificity, without necessitat ing the application of external labels [23]. We present qualitative and quantitative observations regarding the pathway of electromediated molecules entry into giant liposomes as a function of the applied electric pulses. We address the following questions: (i) is it possible to detect membrane permeabilization (assessed by sucrose and glucose mixing) simply by using phase contrast microscopy? (ii) what is the effect of electric pulses parameters on membrane permeabilization? (iii) how does the size of vesicles control/affect the response of the electric field? (iv) can the CARS be used to visualize membrane changes associ ated to permeabilization?

\section{Materials and methods}

\subsection{Materials}

The lipid used to prepare giant unilamellar vesicles was: 1,2 dioleoyl sn glycero 3 phosphocholine (DOPC) (from Avanti Polar Lipids, Alabaster, Alabama, US). The lipids were stored in chloroform at $-20^{\circ} \mathrm{C}$.

\subsection{Preparation of giant unilamellar vesicles}

Giant unilamellar vesicles (GUVs) were produced by the electrofor mation method [17,24]. A $500 \mu \mathrm{g} / \mathrm{mL}$ lipid chloroform solution with the lipid of interest was prepared. Fifteen microliters of this solution were spread homogeneously on the surface of two conductive glass slides coated with indium tin oxide (Sigma). Two strips of adhesive copper were placed on each conductive side of the glass slides. These glasses were placed under vacuum for about $2 \mathrm{~h}$ to evaporate all the organic solvent, and then arranged with their conductive sides facing each other. They were separated by a silicon frame to form a sealed chamber into which the formation buffer ( $240 \mathrm{mM}$ sucrose) was inserted. The electroformation temperature was above the phase transition tempera ture of the lipid composing GUVs. The two glasses were connected to a low voltage Exact ${ }^{\circledR}$ generator (model 128 , Hillsboro, OR) via the copper strips, the chamber was then filled with a solution of $240 \mathrm{mM}$ sucrose, and a sinusoidal current of $25 \mathrm{mV}$ with a frequency of $8 \mathrm{~Hz}$ was applied. The voltage was gradually increased to $1225 \mathrm{mV}$ in $100 \mathrm{mV}$ steps every $5 \mathrm{~min}$. The chamber was then kept at the formation temperature and at $1225 \mathrm{mV}$ with a frequency of $8 \mathrm{~Hz}$ overnight. To peel off the vesicles from the glasses, square wave DC pulses at $1225 \mathrm{mV}$ with a frequency of $4 \mathrm{~Hz}$ were applied. The vesicle solution was then put in an Eppendorf tube and stored at $4{ }^{\circ} \mathrm{C}$.

\subsection{Preparation of the chamber for giant unilamellar vesicles}

The observation chamber consisted of a glass slide and a coverslip [17]. On the glass, a pair of parallel electrodes composed of two strips of adhesive copper were placed $5 \mathrm{~mm}$ apart. Two layers of parafilm were placed on each of the strips and a coverslip was placed on top. Then, the observation chamber was heated to $200{ }^{\circ} \mathrm{C}$ to adhere the coverslip to the glass. The thickness of the chamber was $2 \mathrm{~mm}$ and the distance of the electrodes from the glass substrate was less than $1 \mathrm{~mm}$.

\subsection{Phase contrast observation and pulsation of GUVs}

GUVS were visualized using an inverted Leica DM IRB® microscope (Wetzlar, Germany) microscope equipped with a $40 \times$ objective. Images were taken with a Quantem 512SC $®$ digital camera (Roper Scientific, Germany) mounted on the microscope and connected to a computer. Sample illumination was achieved by phase contrast with a halogen lamp. $5 \mu \mathrm{l}$ of the vesicle solution was put inside the chamber and was then diluted with the pulsing buffer. The pulsing buffers $(\mathrm{pH}=7.35)$ were composed of $260 \mathrm{mM}$ glucose, $1 \mathrm{mM} \mathrm{NaCl}, 1 \mathrm{mM}$ HEPES for phase contrast microscopy and of $1 \mathrm{mM} \mathrm{NaCl}$ in water for CARS micros copy. The osmolarities were $240 \mathrm{mOsm} / \mathrm{kg}$ for the formation medium, and 262 for the pulsation buffer. Conductivities of internal and external solutions were respectively 17 and $260 \mu \mathrm{S} / \mathrm{cm}$. The small difference in osmolarities ensured that the GUVs, chosen with a narrow size distribu tion, have a reproducible and homogeneous initial membrane tension, and the low conductivities prevented Joule heating in the pulsation chamber. The osmotic pressure was the same for all GUVs inducing the same membrane tension [25]. The sugar asymmetry yielded a den sity difference (sucrose is heavier than glucose) that allowed sedimen tation of the vesicles to the bottom of the chamber and facilitated their localization. Moreover, it was used in this work to detect membrane permeabilization by content mixing and loss of contrast. About 5 min after filling the GUV solution into the chamber, the vesicles were mostly located at the bottom of the chamber due to gravity. As reported by the group of Kinosita, GUVs were prepared in an internal medium containing sucrose and diluted in the external medium containing glucose. Since the refractive index of a sucrose solution is higher than that of a glucose so lution, the internal part of the liposomes looked darker than the outside. The quantitative intensity change, detected by phase contrast optics, was used as a measure of the changing sucrose glucose ratio inside the vesicle $[20,26]$.

The chamber was connected to a high voltage unipolar $\beta$ tech ${ }^{\circledR S} 20 \mathrm{u}$ generator (l'Union, France) which delivered square wave DC pulses. The vesicles were pulsed with different electric parameters: number of pulses, duration of the pulse and intensity. The shape of the pulses was checked with an oscilloscope. GUVs were chosen to be at equal dis tance of the two electrodes where the electric field was homogeneous [27]. They were diluted to avoid field inhomogeneity linked to vesicle crowding [28]. Measurements on different vesicles were performed in a new chamber for each condition. Images were acquired and analyzed 
by the Metavue $($ software with an exposure time of $25 \mathrm{~ms}$, just before the application of the pulse train and a few seconds after it.

\subsection{Data processing for the determination of the vesicles sizes on phase contrast pictures}

The sequence of data acquisition was as follows. One image before and one after pulsing were recorded for each vesicle with no binning. Data processing was done with the Imagej® Software. A median filter at 2 pixels was applied on the images to reduce the shot noise while keeping the resolution very high. The intensity was assessed using the brightness profile along the line across the vesicle in a radial direction. Lipid membrane was detected by two intensity peaks which distance determined the vesicle diameter (Fig. 1B). Under our experi mental conditions, 2 pixels represent $1 \mu \mathrm{m}$. As a median 2 pixels filter was applied, this limit of a change of $1 \mu \mathrm{m}$ in GUVs diameter was within the limit of optical resolution. This represents a detectable change of about $3 \%$ of the GUVs size for GUVs around $30 \mu \mathrm{m}$ in diameter.

\subsection{CARS microscopy}

The microscope setup is based on a Leica TCS SP5 multiphoton microscope. A Leica $0.85 \mathrm{NA}, 40 \times$ air objective was employed to record data in transmission. CARS signals were collected by a Leica $0.55 \mathrm{NA}$ air condenser and transmitted through suitable emission filters (641/75 and 775/SP, Semrock, United States). A Nd:YVO4 laser delivering $4 \mathrm{~W}$ of $7 \mathrm{ps}$ pulses at a repetition rate of $76 \mathrm{MHz}$ and a wavelength of $532 \mathrm{~nm}$ (HighQ Laser, Austria) was used to pump an optical parametric oscillator (APE, Germany) the signal beam of which was used as a pump beam [23], whereas residual pump light from the Nd:YV4 laser was used as Stockes beam. Images were recorded at the aliphatic C $\mathrm{H}$ vibra tional resonance of $2845 \mathrm{~cm}^{-1}$.

\section{Results}

We performed experiments regarding the effects of electric pulses on giant unilamellar vesicles. We used phase contrast and CARS micros copies with the aim to gain insight the electropermeabilization phe nomena on pure lipid systems. Differences in refraction index of the two media inside and outside the GUVs permit GUVs to be easily ob served using phase contrast. The use of fluorescent lipid analogs, in the membrane of GUVs, or the use of fluorescent dyes in the formation or pulsation medium, to detect molecule exchange between the GUVs and the external medium, was therefore not mandatory. Membrane permeabilization was assessed by a decrease in contrast between GUVs and the external medium. Membrane associated alterations, resulting in lipid loss and a subsequent decrease in diameter, were ob served by phase contrast and CARS. 10 pulses were applied at a rate of $1 \mathrm{~Hz}$. The durations of the applied pulses were $100 \mu \mathrm{s}$ or $5 \mathrm{~ms}$, and are similar to those used for electromediated cytotoxic drug loading and gene electrotransfer [29]. The delay between pulses was also chosen to be similar to the ones used in drugs and genes delivery. It varied be tween 1 and $2 \mathrm{~s}$. Under those conditions, previous published studies showed that lipid bilayer resealing was achieved during the interpulse delay $[16,30]$.

\subsection{GUVs permeabilization is detected by using phase contrast microscopy}

GUVs were firstly observed by phase contrast microscopy here in Fig. 1. As described above, the presence of sucrose in the solution used to prepare the vesicles suspension and glucose in the solution used to pulse the vesicles allows their direct observation (Fig, 1A). GUVs were submitted to $5 \mathrm{~ms}$ electric pulses, applied at $0.7 \mathrm{kV} / \mathrm{cm}$ in tensity. The total number of pulses was 10 . Images were taken before the train of pulses and after each pulse. Results reported in Fig. 1A, show that increasing the number of pulses results in a decrease in
A

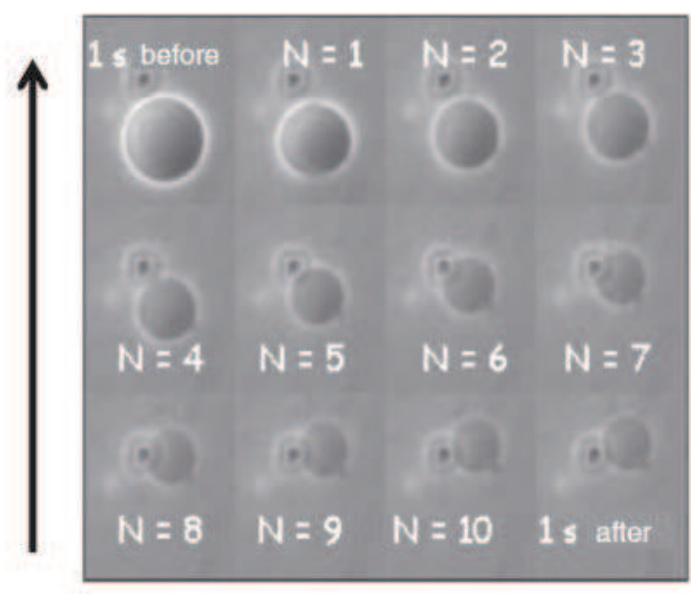

B

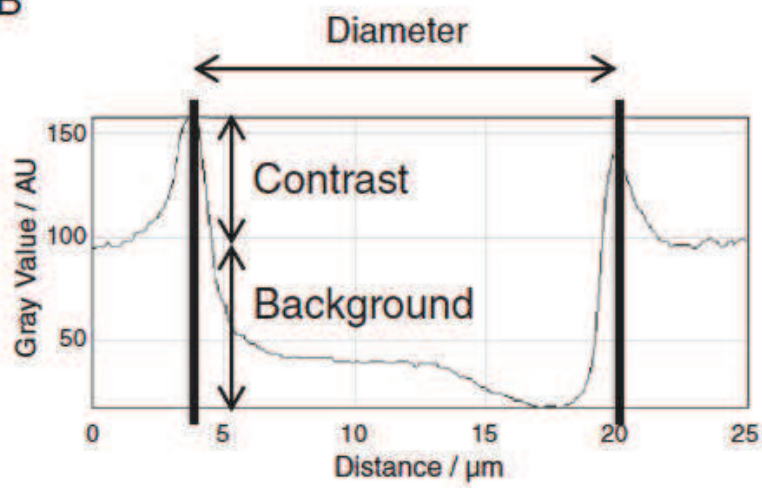

C

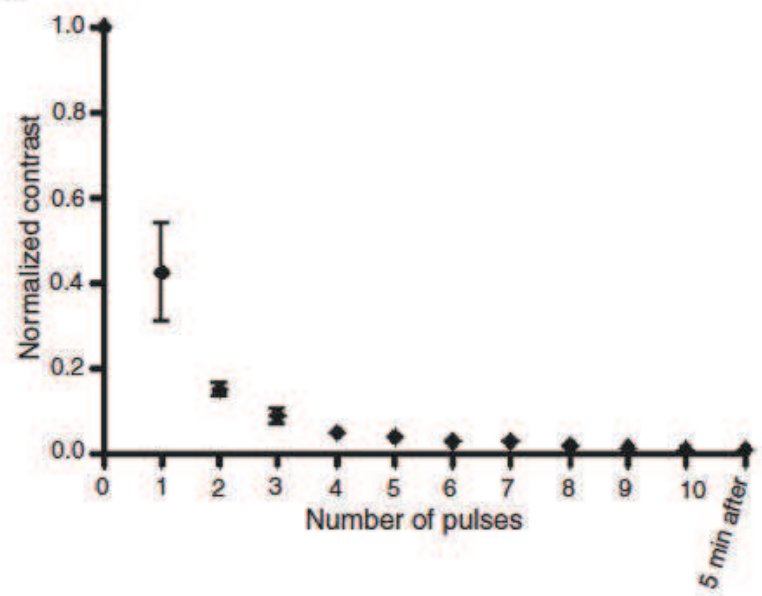

Fig. 1. Effect of the number of pulses on the contrast of GUVs. (A) Pictures of GUVs submitted to an increasing number of pulses. (B) Plot profile of the GUV. Diameter was evaluated by measuring the distance between the two peaks. Background is constant for each picture. Contrast was evaluated by measuring the intensity of each peak above the background. (C) Evolution of the contrast of GUVs as a function of the number of pulses. The more pulses are applied to GUVs, the more their contrast is lost. The black arrow indicates the electric field direction.

contrast. This decrease was detected as soon as the first pulse was ap plied and was enhanced with an increase in pulse number. One has to notice that the loss of contrast was a fast process occurring in less than $1 \mathrm{~s}$ after pulse delivery (see Supplementary data) in agreement with previous works $[17,31]$. After 10 pulses, a difference of contrast was still present allowing the easy observation of the GUV. This dif ference remained the same just after the 10th pulse and a few sec onds later. Under these experimental conditions, with a $1 \mathrm{~s}$ delay 
between successive pulses, decrease in contrast, when present, was a consequence of pulse delivery.

Light plot profiles (Fig. 1B) were created from these images in order to quantify the decrease in contrast. As shown in Fig. 1C, the decrease in contrast was triggered by the first pulse to be further en hanced with the following ones.

\subsection{GUVs permeabilization is associated to lipid loss}

Decrease in contrast was indeed also associated to lipid loss. We therefore assess the role of the number of applied pulses on this lipid loss. For this purpose, GUVs were submitted to $5 \mathrm{~ms}$ electric pulses, applied at a $1 \mathrm{~Hz}$ frequency and at $0.7 \mathrm{kV} / \mathrm{cm}$ intensity (as reported in Fig. 1A). The total number of pulses was 10 . Images were taken before and after each pulse application. Results reported in Fig. 2, show that in creasing the number of pulses results in a decrease in GUV sizes. This decrease was detected as soon as the first pulse was applied and was enhanced with an increase in the number of pulses. It reached $40 \%$ after 10 pulses. This experiment, performed on GUVs with different diameters, was reproducible. There is a good correlation between the decrease in size and the decrease in contrast (see Fig. 1C).

\subsection{GUVs permeabilization is under the control of electric field strength}

The decrease of the GUVs size was then used to study the effect of the field intensity. 10 pulses were applied with duration equal to $100 \mu$ s or $5 \mathrm{~ms}$. For electric pulses lasting $100 \mu \mathrm{s}$, a $1360 \mathrm{~V} / \mathrm{cm}$ electric field led to a 5\% decrease in size of the largest vesicle (diameter around $40 \mu \mathrm{m}$ ) with no effect on the smaller one (diameter around $30 \mu \mathrm{m}$ ) (Fig. 3A). A further increase in the field strength to $1420 \mathrm{~V} / \mathrm{cm}$ resulted in a $10 \%$ size decrease of the smaller vesicles, with a concomitant de crease of the larger one. The $5 \mathrm{~ms}$ pulse durations were observed to ex haust these observations (Fig. 3B). Pulses applied at $210 \mathrm{~V} / \mathrm{cm}$ were observed to induce the decrease in size of the GUV with diameter around $40 \mu \mathrm{m}$. Once again, the largest vesicles being affected for lower fields than the smaller ones. Increasing the field to $260 \mathrm{~V} / \mathrm{cm}$ led to the decrease in size of the smallest GUVs (diameter around $20 \mu \mathrm{m}$ ) and was associated to a strong decrease in contrast. The threshold for membrane destabilization, i.e. the electric field value leading to lipid loss, has been determined for both pulses duration as a function of GUVs size. GUVs diameter was observed to be affected for electric field values $\mathrm{E}$ above a threshold $\mathrm{E}_{\mathrm{c}}$. This threshold was defined as the minimum electric field value leading to a significant decrease (i.e. $1 \mu \mathrm{m})$ in GUVs diameter. It is an overestimation of the "real" threshold

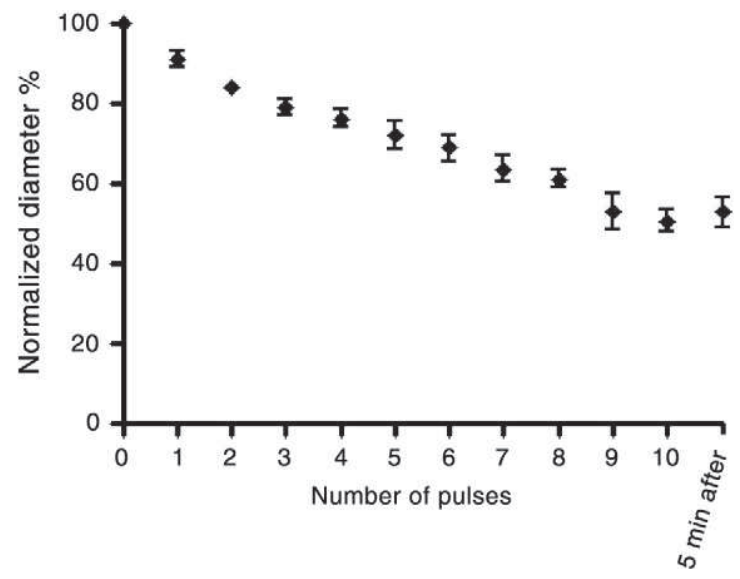

Fig. 2. Evolution of the GUVs size decrease as a function of the number of pulses. The more pulses are applied to GUVs, the more their size decrease. GUVs diameters were measured from the light plot profile (Fig. 1-B) done on the images of 5 GUVs with initial diameters varying from 10 to $40 \mu \mathrm{m}$. value due to the poor sensitivity of the method of detection, the use of flow of small ions inside the GUVs being the most sensitive method (manuscript under preparation). That value depended on the size of the vesicle, the largest vesicles being affected for the lowest field inten sities. As shown in Fig. 3C, there was a linear relationship between $E_{c}$ and the radius of the GUVs.

\subsection{GUVs permeabilization assessed by CARS microscopy}

In previous works, we reported that the decrease in size on the GUVs was due to ejection of lipid material in the form of small vesi cles and tubules [17]. However this lipid ejection causing the vesicle radius to diminish was visualized by the use of fluorescent lipid ana logs inserted into the membrane of the GUVs. It could therefore rep resent a label induced artifact. Our objective therefore was to prove that electropermeabilization, due to harsh electrical conditions, is as sociated to lipid loss. For that, we used DOPC vesicles and submitted them to a series of 20 pulses lasting $5 \mathrm{~ms}$ at a $1 \mathrm{~Hz}$ frequency. The field intensity was chosen in order to induce lipid loss. CARS micros copy was used as a label free imaging tool. As can be seen in Fig. 4 and in the associated movie, it is possible to optically detect the effects of electropermeabilization of GUVs made with pure lipids here in Fig. 4. The images show vesicles exhibiting lipid loss via formation of tubu lar structures. These structures were created on the electrodes facing side of the GUV, and appeared to remain attached to the vesicle and stable over a few minutes, as previously reported by using fluores cence microscopy.

\section{Discussion and conclusion}

The objectives of our works were to analyse the relevance of GUVs as a simple and convenient model to study membrane electroper meabilization phenomena. Imaging was used in order to get a direct access to the underlying consequences of pulse application. No addi tion of exogenous molecules (lipid analogue, fluorescent dye) was re quired in order to perform the analysis on pure lipid systems thus excluding possible artefacts linked to these molecules. We addressed two questions: i) the effect of electric pulses parameters on mem brane permeabilization and ii) the associated membrane alteration, lipid loss resulting in a decrease in size of the permeabilized vesicles. For that, our strategy consisted on using phase contrast microscopy to detect content mixing and CARS microscopy to visualize membrane alterations.

It is well known, for many years, that the trigger of membrane electropermeabilization is the transmembrane potential which must reach critical values between $200 \mathrm{mV}$ and $1 \mathrm{~V}$ [21,22,32], which have been determined in vesicles as well as on bacteria, plant and mammalian cells. The application of the electric field indeed superim poses an electro induced transmembrane potential, $\Delta \psi_{\mathrm{i}(\mathrm{M})}$, to the resting transmembrane potential $\Delta \psi_{0}$. The value of $\Delta \psi_{\mathrm{i}}$ is given by the Schwan equation:

$\Delta \psi_{i(M)} \quad \boldsymbol{f} \cdot \mathbf{g}(\boldsymbol{\lambda}) \cdot \boldsymbol{r} \cdot \boldsymbol{E} \cdot \cos (\boldsymbol{\theta}(\boldsymbol{M}))$

where $f$ is a factor depending on the vesicle shape, $g(\lambda)$ a parameter depending on the conductivities $\lambda$ of the membrane, of the external and internal media, $r$ is the radius of the vesicle, $E$ the electric field strength, and $\theta(\mathrm{M})$ the angle between the electric field direction and the normal to the membrane at the considered point $\mathrm{M}$ of the surface. Being dependent on the angle $\theta(\mathrm{M})$, the electric field effect is not uniform along the cell membrane and its maximum effects are present at the poles of the cells facing the electrodes. This is of course is valid as long as the membrane conductivity remains very low. Apart from pulse strength E, pulse number and pulse duration are another key parameters for the success of permeabilization, 
A
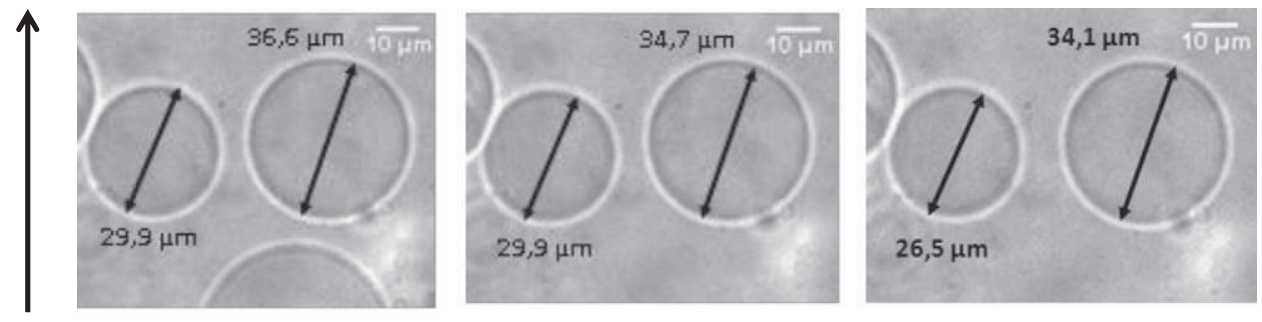

B
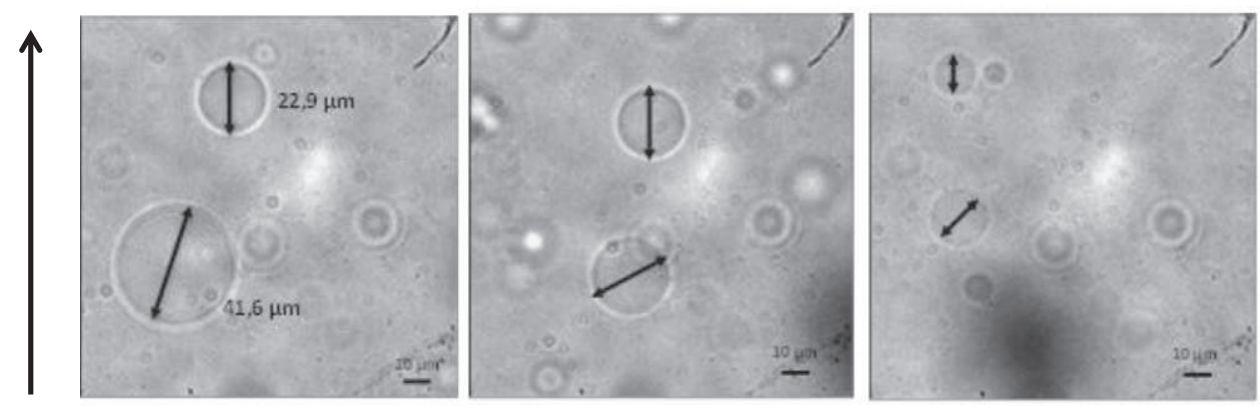

C

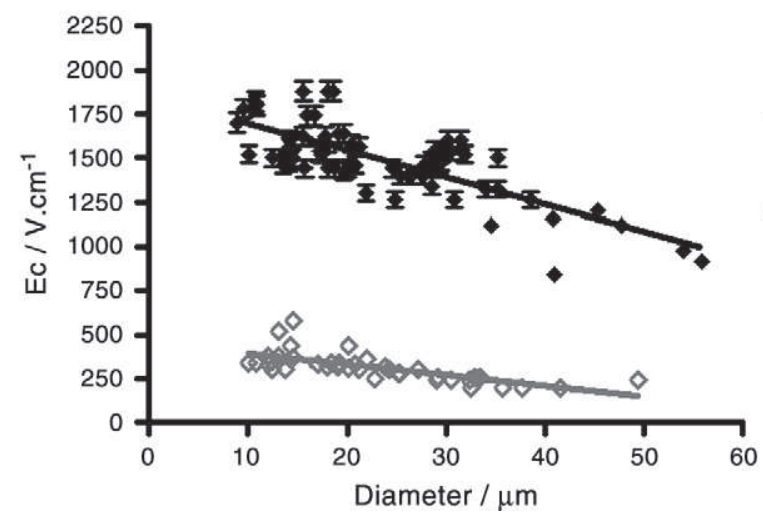

- 10 pulses of duration $100 \mu$ s at $1 \mathrm{~Hz}$

- 10 pulses of duration $5 \mathrm{~ms}$ at $1 \mathrm{~Hz}$

Fig. 3. Effect of an increasing electric field strength on GUVs with different sizes. 10 pulses at $1 \mathrm{~Hz}$ of $100 \mu$ s duration (A) or $5 \mathrm{~ms}$ duration (B) are applied. Left pictures (A and B) were taken before the application of electric pulses. (A) In the middle: size decrease of the larger GUVs after application of a $1360 \mathrm{~V} / \mathrm{cm}$ electric field. On the right: size decrease of the smaller GUVs after application of a $1420 \mathrm{~V} / \mathrm{cm}$ electric field. (B) In the middle: size decrease of the larger GUVs after application of a $210 \mathrm{~V} / \mathrm{cm}$ electric field. In the right: size decrease of the two GUVs after application of a $260 \mathrm{~V} / \mathrm{cm}$ electric field. (C) Evolution of the threshold value for permeabilization as a function of the initial diameter of GUVs. The black arrow indicates the electric field direction.

especially in the case of large molecules such as plasmid DNA which are driven by electrophoresis towards the permeabilized membrane [33].

Electric field parameters (field strength, number and duration) were therefore varied in order to study their effect. The basics for electroper meabilization imply that the transmembrane potential must reach values close to $200 \mathrm{mV}$. According to Eq. (1), electric field strength values $\mathrm{E}$ higher than a threshold value $\mathrm{E}_{\mathrm{c}}$ must be applied to induce membrane permeabilization. In the case of two vesicles 1 and 2 with radii $r_{1}$ and $r_{2}$, respectively, permeabilization should be induced for a constant Ec $r$ value. This implies that if $r_{1}>r_{2}$, then $\mathrm{Ec}_{1}<\mathrm{Ec}_{2}$.

As shown in Fig. 3, this clearly is the case. Large vesicles are affected by electric field values which do not affect the smaller vesicles. There is a direct linear relationship between $\mathrm{E}_{\mathrm{c}}$ and vesicle radius (Fig. 1D). More over, the larger the vesicles are, the lower the electric field threshold value is. One however has to notice that the determination of the "thresh old values" $E_{c}$ by the method used here, contrast decrease, is not a very sensitive one. Our recent works indeed show that the detection method is a very important parameter for the precise determination of the critical transmembrane potential. As fluorescence is more sensitive, the use of quenching ions as probes for lipid bilayer permeabilization allows very high resolution detection because of their small size which implies the need for a lower membrane destabilization than the one needed with larger molecules such as fluorescent dyes or sugar (submitted manuscript).

We also studied the effects of the number of cumulated pulses and of their duration. As for cells, both parameters control the process. Increasing the number of pulses from 1 to 20 increases membrane permeabilization as observed by molecule exchange and liposome size decrease and, for longer pulses duration, tubule formation. Once again, these experimental data are similar to those obtained with mam malian cells. Altogether these results lead to the conclusion that GUVs are a good and relevant model to study membrane alteration under electric field application. This system presents the characteristic of having no cytoskeleton. Dramatic membrane consequences can there fore be observed such as lipid loss and tubule formation. Up to now, they could be only detected by using fluorescence microscopy. We pre sent here for the first time results of electropermeabilization of pure 


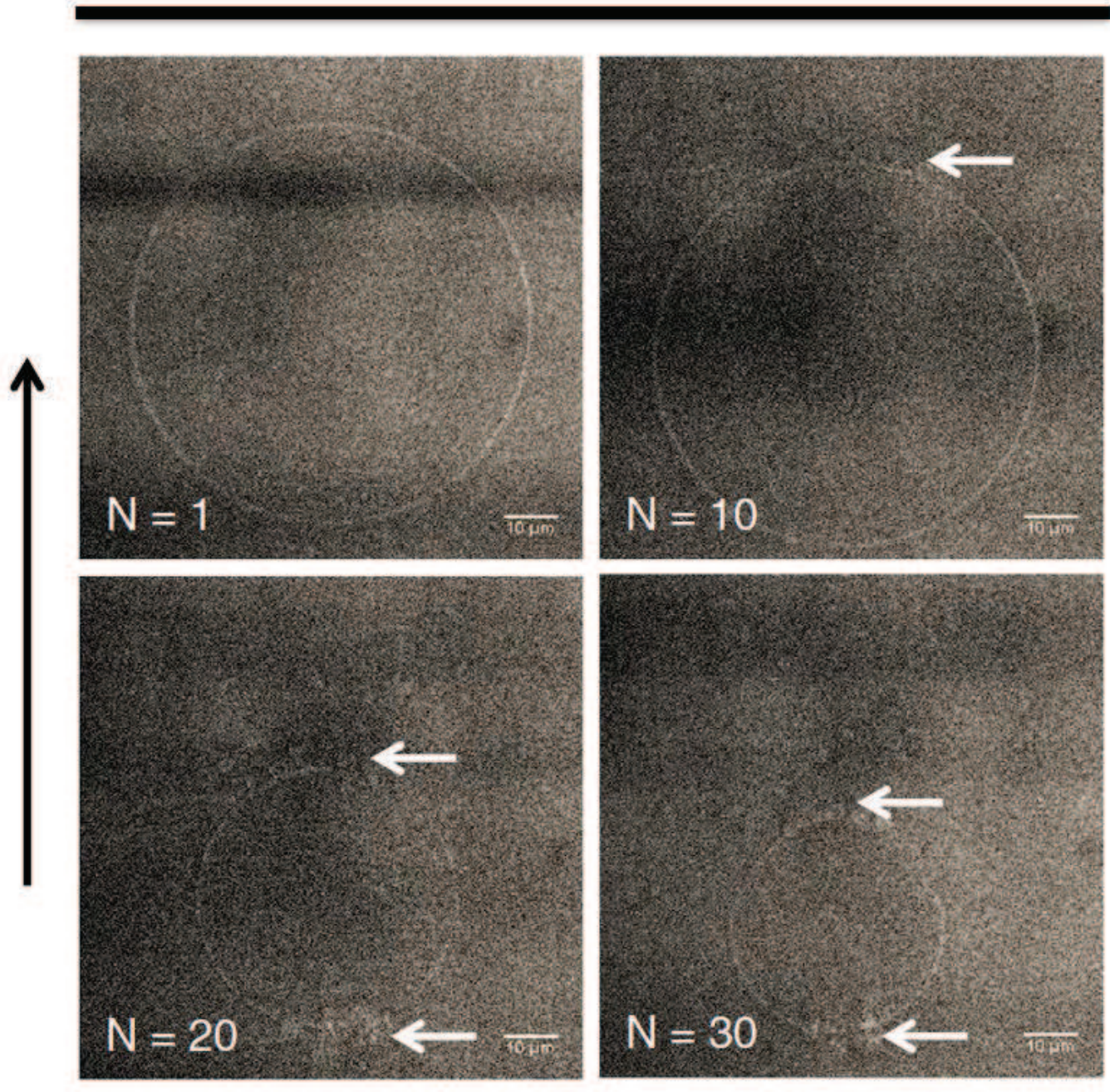

Fig. 4. Visualization by CARS microscopy of GUV electropermeabilization. Images 1, 10, 20 and 30 of a time series acquired during electropermeabilization with an interval between each image of $310 \mathrm{~ms}$. Image size: $151.17^{*} 151^{*} 17 \mu \mathrm{m}$ with a resolution of $295 \mathrm{~nm} /$ pixel. White arrows indicate nanotubes shed from GUVs upon electropermeabilization. The black arrow indicates the electric field direction (see supplementary movie).

lipid systems obtained by CARS microscopy. Optical microscopy is unique in its ability to probe living specimens with subcellular resolu tion. Fluorescence microscopy, while extremely sensitive and widely used, requires the introduction of extrinsic fluorophores but often causes unwanted perturbations. Strong vibrational signals can be obtained with CARS microscopy, a nonlinear Raman technique. Our results indicate that tubule formation indeed is not an artifact due to fluorescence labeling. Similar results are obtained for both fluorescence and CARS microscopy. However, such an experimental approach has a limitation, namely lack of molecular specificity which is critical when complex lipid mixtures are studied (CARS microscopy is suitable only for following the fate of a major membrane component).

As a conclusion, these results underline the high potential of phase contrast and CARS microscopies as a tool to study electric field effects in living systems

\section{Acknowledgements}

We wish to thank Emilie Phez, Jean Michel Escoffre, Cyril Favard and lan Huerts for their participation in the inception of this work. This work has been supported by PROCOPE (collaboration between the group of Marie Pierre Rols and Andreas Zumbusch), the AFM and the ANR Cemibio and was conducted in the scope of the EBAM European Associated Laboratory (LEA). Imaging experiments were run at the TRI platform (funded by the region Midi Pyrenees, the grand Toulouse cluster and the EU FEDER),

\section{References}

[1] S. Hacein-Bey-Abina, C. Von Kalle, M. Schmidt, M.P. McCormack, N. Wulffraat, P. Leboulch, A. Lim, CS. Osborne, R. Pawliuk, E. Morillon, R. Sorensen, A. Forster, P. Fraser, J.I. Cohen, G. de Saint Basile, L. Alexander, U. Wintergerst, T. Frebourg. A. Aurias, D. Stoppa-Lyonnet, S. Romana, I. Radford-Weiss, F. Gross, F. Valensi, E. Delabesse, E. Macintyre, F. Sigaux, J. Soulier, L.E. Leiva, M. Wissler, C Prinz, T.H Rabbitts, F. Le Deist, A. Fischer, M. Cavazzana-Calvo, LMO2-associated clonal T cell proliferation in two patients after gene therapy for SCID-X1, Science 302 (2003) 415-419,

[2] M.P. Rols, Gene transfer by electrical fields, Curr. Gene Ther, 10 (2010) 255.

[3] F.M. Andre, LM. Mir, Nucleic acids electrotransfer in vivo: mechanisms and practical aspects, Curr. Gene Ther. 10 (2010) 267-280.

[4] E. Neumann, K. Rosenheck, Permeability changes induced by electric impulses in vesicular membranes, J. Membr. Biol. 10 (1972) 279-290.

[5] L.M. Mir, LF. Glass, G. Sersa, J. Teissie, C. Domenge, D. Miklavcic, M.]. Jaroszeski, S. Orlowski, D.S. Reintgen, Z. Rudolf, M. Belehradek, R. Gilbert, M. Rols, J. Belehradek Jr., JM. Bachaud, R DeConti, B. Stabuc, M. Cemazar, P. Coninx, R. Heller, Effective treatment of cutaneous and subcutaneous malignant tumours by electrochemotherapy, Br.J. Cancer 77 (1998) 2336-2342

[6] G. Sersa, D. Miklavcic, M. Cemazar, Z. Rudolf, G. Pucihar, M. Snoj, Electrochemotherapy in treatment of tumours, Eur. J. Surg. Oncol. 34 (2008) 232-240.

[7] J. Gehl, Electroporation for drug and gene delivery in the clinic: doctors go electric, Methods Mol. Biol. 423 (2008) 351-359. 
[8] M. Cemazar, T. Jarm, G. Sersa, Cancer electrogene therapy with interleukin-12, Curr. Gene Ther. 10 (2010) 300-311.

[9] A.I. Daud, R.C. DeConti, S. Andrews, P. Urbas, A.I. Riker, V.K. Sondak, P.N. Munster, D.M. Sullivan, K.E. Ugen, J.L. Messina, R. Heller, Phase I trial of interleukin-12 plasmid electroporation in patients with metastatic melanoma, J. Clin. Oncol. 26 (2008) 5896-5903.

[10] J. Teissie, M. Golzio, M.P. Rols, Mechanisms of cell membrane electropermeabilization: a minireview of our present (lack of ?) knowledge, Biochim. Biophys. Acta 1724 (2005) 270-280.

[11] J.M. Escoffre, T. Portet, C. Favard, J. Teissie, D.S. Dean, M.P. Rols, Electromediated formation of DNA complexes with cell membranes and its consequences for gene delivery, Biochim. Biophys. Acta 1808 (2011) 1538-1543.

[12] A. Paganin-Gioanni, E. Bellard, J.M. Escoffre, M.P. Rols, J. Teissie, M. Golzio, Direct visualization at the single-cell level of siRNA electrotransfer into cancer cells, Proc. Natl. Acad. Sci. U. S. A. 108 (2011) 10443-10447.

[13] K.A. Riske, R. Dimova, Electric pulses induce cylindrical deformations on giant vesicles in salt solutions, Biophys. J. 91 (2006) 1778-1786.

[14] C.K. Haluska, K.A. Riske, V. Marchi-Artzner, J.M. Lehn, R. Lipowsky, R. Dimova, Time scales of membrane fusion revealed by direct imaging of vesicle fusion with high temporal resolution, Proc. Natl. Acad. Sci. U. S. A. 103 (2006) 15841-15846.

[15] J. Teissie, T.Y. Tsong, Electric field induced transient pores in phospholipid bilayer vesicles, Biochemistry 20 (1981) 1548-1554.

[16] E. Tekle, R.D. Astumian, W.A. Friauf, P.B. Chock, Asymmetric pore distribution and loss of membrane lipid in electroporated DOPC vesicles, Biophys. J. 81 (2001) 960-968.

[17] T. Portet, F. Camps i Febrer, J.M. Escoffre, C. Favard, M.P. Rols, D.S. Dean, Visualization of membrane loss during the shrinkage of giant vesicles under electropulsation, Biophys. J. 96 (2009) 4109-4121.

[18] L.V. Chernomordik, A.V. Sokolov, V.G. Budker, Electrostimulated uptake of DNA by liposomes, Biochim. Biophys. Acta 1024 (1990) 179-183.

[19] T. Portet, C. Favard, J. Teissie, D. Dean, M.P. Rols, Insights into the mechanisms of electromediated gene delivery and application to the loading of giant vesicles with negatively charged macromolecules, Soft Matter 7 (2011) 3872-3881.
[20] K. Akashi, H. Miyata, H. Itoh, K. Kinosita Jr., Preparation of giant liposomes in physiological conditions and their characterization under an optical microscope, Biophys. J. 71 (1996) 3242-3250.

[21] K.A. Riske, R. Dimova, Electro-deformation and poration of giant vesicles viewed with high temporal resolution, Biophys. J. 88 (2005) 1143-1155.

[22] D.V. Zhelev, D. Needham, Tension-stabilized pores in giant vesicles: determination of pore size and pore line tension, Biochim. Biophys. Acta 1147 (1993) 89-104.

[23] M. Muller, A. Zumbusch, Coherent anti-Stokes Raman Scattering Microscopy, Chemphyschem 8 (2007) 2156-2170.

[24] M.I. Angelova, D.S. Dimitrov, Liposome electroformation, Faraday Discuss. Chem Soc. 81 (1986) 303-311.

[25] E. Evans, Entropy-driven tension in vesicle membranes and unbinding of adherent vesicles, Langmuir 7 (1991) 1900-1908

[26] L. Kristanc, S. Svetina, G. Gomiscek, Effects of the pore-forming agent nystatin on giant phospholipid vesicles, Biochim. Biophys. Acta 1818 (2012) 636-644.

[27] S. Mazeres, D. Sel, M. Golzio, G. Pucihar, Y. Tamzali, D. Miklavcic, J. Teissie, Non invasive contact electrodes for in vivo localized cutaneous electropulsation and associated drug and nucleic acid delivery, J. Control. Release 134 (2009) 125-131.

[28] G. Pucihar, T. Kotnik, J. Teissie, D. Miklavcic, Electropermeabilization of dense cell suspensions, Eur. Biophys. J. 36 (2007) 173-185.

[29] M. Golzio, J.M. Escoffre, T. Portet, C. Mauroy, J. Teissie, D.S. Dean, M.P. Rols, Observations of the mechanisms of electromediated DNA uptake-from vesicles to tissues, Curr. Gene Ther. 10 (2010) 256-266.

[30] S. Raffy, J. Teissie, Electroinsertion of glycophorin A in interdigitation-fusion giant unilamellar lipid vesicles, J. Biol. Chem. 272 (1997) 25524-25530.

[31] T. Portet, R. Dimova, A new method for measuring edge tensions and stability of lipid bilayers: effect of membrane composition, Biophys. J. 99 (2010) 3264-3273.

[32] J. Teissie, M.P. Rols, An experimental evaluation of the critical potential difference inducing cell membrane electropermeabilization, Biophys. J. 65 (1993) 409-413.

[33] M.P. Rols, J. Teissie, Electropermeabilization of mammalian cells. Quantitative analysis of the phenomenon, Biophys. J. 58 (1990) 1089-1098. 